\title{
Communication
}

\section{Drug-Induced Liver Injury Predictions: Extended Clearance Model and Its Use for Prospective Transporter and Enzyme-Based Hepatic Cell Stress Grading}

\section{Gian Camenisch}

Department of PK Sciences, Novartis Institutes for BioMedical Research, Basel, Switzerland

Email address:

gian.camenisch@novartis.com

\section{To cite this article:}

Gian Camenisch. Drug-Induced Liver Injury Predictions: Extended Clearance Model and Its Use for Prospective Transporter and Enzyme-Based Hepatic Cell Stress Grading. International Journal of Pharmacy and Chemistry. Vol. 5, No. 2, 2019, pp. 15-19. doi: $10.11648 /$ j.jpp. 20190502.11

Received: May 24, 2019; Accepted: June 27, 2019; Published: July 9, 2019

\begin{abstract}
Many enzymes and transporters involved in the hepatic clearance of drugs also play an important role in endogenous compound transport. Inhibition of some of these active mechanisms has frequently been shown to be associated with Drug-Induced Liver Injury (DILI). The Extended Clearance Model (ECM) describes the complex interplay between the different processes driving hepatic clearance, namely sinusoidal uptake and efflux, canalicular secretion and intracellular metabolism. Based on the ECM, we have derived an integral concept (referred as 1/R-value approach) to quantitatively describe the overall inhibition potency of potential drug candidates on active processes involved in the transport and metabolism of endogenous and safety-relevant compounds. For a small training set of in-house compounds with largely complete in vitro inhibition and in vivo exposure data, accurate ECM-based prediction of DILI was realized. Additionally, prediction of several cases of DILI for a comprehensive validation set of external compounds was achieved with no major false-positive results. However, due to general incompleteness of the required input information available in the public space (the most probable reason for the large number of false-negatives in the test set) the overall legitimacy of ECM for large-scale prediction of cell stress mediated DILI still needs to be demonstrated. In order to advance and accelerate science in this exciting but complex field, a more transparent and open sharing of data is therefore urgently needed and should be encouraged.
\end{abstract}

Keywords: DILI, ECM, Cell Stress, Enzyme and Transporter Inhibition, BDDCS

\section{Introduction}

DILI is defined as liver injury caused by xenobiotics resulting in liver test abnormalities or liver dysfunction. Drug compounds and/or their (reactive) metabolites can cause DILI via cell stress (i.e. directly via exertion of an (undesired) pharmacological effect or indirectly via inhibition of transporters and/or enzymes involved in endogenous drug transport), via hypersensitivity (i.e. due to an immune reaction) and/or due to mitochondrial function impairment [1]. The severity of DILI varies greatly and can hardly be linked to a single symptom or laboratory abnormality. Not surprisingly, various DILI grading systems exist in literature. In dependence on Health Authority proposals, Table 1 provides the severity-grading tactic we have applied for the present analysis [2].

In order to enter liver cells, endo- and exogenous compounds first have to overcome the hepatocyte membrane barrier separating the blood in the sinusoid from the cytosol of the hepatocytes. Uptake across this barrier might occur by passive diffusion and/or, particularly for more hydrophilic compounds, active carrier-mediated transport. Once in the cytosol, drugs are subject to metabolism, efflux transporter-mediated canalicular/biliary secretion and/or efflux back into the sinusoid. The Extended Clearance Model (ECM) quantitatively describes the complex interplay between the different hepatic clearance pathways as follows [3]: 
Table 1. DILI grading system.

\begin{tabular}{lll}
\hline Severity level & Severity class & DILI manifestation \\
\hline \multirow{3}{*}{ No-mild } & 0 & Absence \\
& 1 & Steatosis \\
& 2 & Cholestasis, Steatohepatitis \\
Moderate & 3 & Liver aminotransferases increase \\
& 4 & Hyperbilirubinaemia \\
& 5 & Jaundice \\
Severe & 6 & Liver necrosis \\
& 7 & Acute liver failure \\
& 8 & Necrosis \\
\hline
\end{tabular}

$$
C L_{\text {int }, \text { all }}=\frac{\left(P S_{\text {upt }, a c t}+P S_{\text {upt }, \text { pas }}\right) \cdot\left(C L_{s e c}+C L_{m e t}\right)}{P S_{\text {eff }, a c t}+P S_{\text {pas }}+C L_{\text {sec }}+C L_{m e t}}
$$

Where, $\mathrm{CL}_{\text {int,all }}$ represents the overall apparent intrinsic hepatic clearance, $\mathrm{PS}_{\text {upt,act }}$ is the intrinsic membrane clearances for basolateral (sinusoidal) transporter-mediated (active) influx, $\mathrm{PS}_{\mathrm{upt} \text {,pas }}$ is the intrinsic membrane clearances for basolateral influx via passive diffusion, $\mathrm{PS}_{\mathrm{eff}, \text { act }}$ is the intrinsic clearance for active efflux back into the blood at the sinusoidal membrane, $\mathrm{PS}_{\text {eff,pas }}$ is the intrinsic clearance for the passive back diffusion into the blood at the sinusoidal membrane, $\mathrm{CL}_{\text {met }}$ is the metabolic intrinsic hepatic clearance, and $\mathrm{CL}_{\mathrm{sec}}$ is the intrinsic membrane clearance for biliary secretion at the canalicular side of hepatocytes (generally assumed to be solely a transporter-mediated process).

The objective of current work was, taking into account in vitro enzyme/transporter inhibition information and in vivo exposure data, to develop a new ECM-based prediction model in order to illustrate its ability to accurately predict the cell stress originating DILI potential of drug candidates independent of the impacted metabolism and/or transport pathway(s).

\section{Method}

\subsection{Model Development}

Many enzymes and transporters, predominantly involved in hepatic uptake, metabolism and/or secretion of drugs, are also involved in endogenous compound transport such as bilirubin or bile salts. Inhibition of some of these processes has been clearly associated with DILI (upstream hepatocyte injury causing dose-dependent cell stress) $[4,5]$. The fraction of any intrinsic enzyme and/or transporter clearance process that can be reversibly and/or irreversibly inhibited by a perpetrator (inhibitor) drug can be expressed as [6]:

$$
\frac{C L_{\text {int }, i}}{C L_{\text {int }}}=\frac{1}{\left(1+\sum \frac{[I]}{K_{i}}\right) \cdot\left(1+\sum \frac{k_{\text {inact }}^{\cdot I I]}}{k_{\text {deg }} \cdot\left(K_{I}+[I]\right)}\right)}
$$

Where, $\mathrm{CL}_{\text {int }}$ and $\mathrm{CL}_{\text {int, } \mathrm{i}}$ refer to any intrinsic clearance in the absence and presence of process inhibitors, respectively. [I] is the concentration of the inhibitor, $\mathrm{K}_{\mathrm{i}}$ is its associated reversible inhibition constant, $\mathrm{K}_{\mathrm{I}}$ is the irreversible inhibition constant, $\mathrm{k}_{\text {inact }}$ is the maximal inactivation rate constant, and $\mathrm{k}_{\mathrm{deg}}$ represents the degradation rate constant for the active process of interest.

Referring to the extended clearance concept according to Eq. (1), assuming passive diffusion is identical for efflux and uptake and is not affected by the inhibitor drug (i.e. $\mathrm{PS}_{\text {eff,pas }}=$ $\mathrm{PS}_{\text {upt,pas }}=\mathrm{PS}_{\text {upt,pas,i }}=\mathrm{PS}_{\text {eff,pas,i }}=\mathrm{PS}_{\mathrm{pas}}$ ), the fraction of total hepatic intrinsic clearance affected by pathway or process inhibition following oral drug administration is represented by [7]:

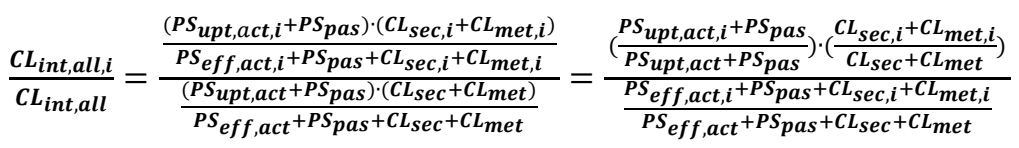

After reorganizing, substituting Eq. (2) into Eq. (3), assuming back-efflux over the sinusoidal membrane to be unaffected by the inhibitor compound (i.e. $\mathrm{PS}_{\text {eff,act,i }}=\mathrm{PS}_{\text {eff,act }}$ ) and supposing absence of irreversible inhibition on active transport processes (i.e. sinusoidal uptake/efflux and canalicular secretion), the above relationship can sufficiently be approximated with $[3,8]$ :

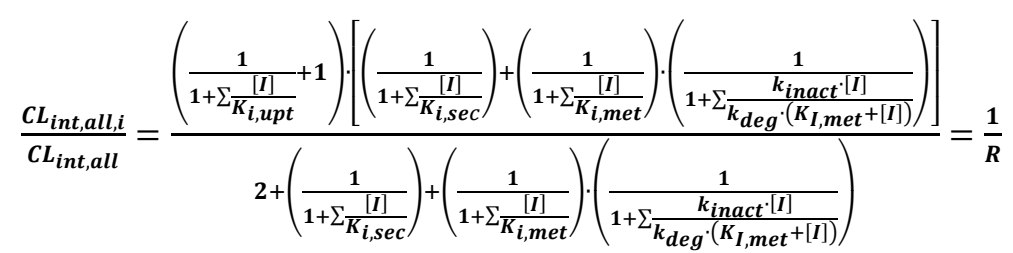

In alignment with pertinent literature in the field of drug-drug interactions the above relationship is denoted as $1 /$ R-value [6]. [I] preferentially refers to the unbound maximum steady-state concentration at the highest efficacious daily dose $\left(\mathrm{C}_{\text {max,ss,u}}\right)$ [4]. Accordingly, for $K_{i}$ and $K_{I}$ the numbers referring to the concentration of the unbound drug in the in vitro test system are used (referred as $\mathrm{K}_{\mathrm{i}, \mathrm{u}}$ and $\mathrm{K}_{\mathrm{I}, \mathrm{u}}$ ).
Consequently, assuming DILI to be a direct inhibitory consequence of sinusoidal uptake (reversible via NTCP, OATP1B1 and/or OATP1B3), canalicular efflux (reversible via BSEP and/or MRP2) and/or enzymatic metabolism (reversible via UGT1A1 and/or irreversible via CYP3A4), Eq. 4 can be rewritten as follows $[4,5]$ : 


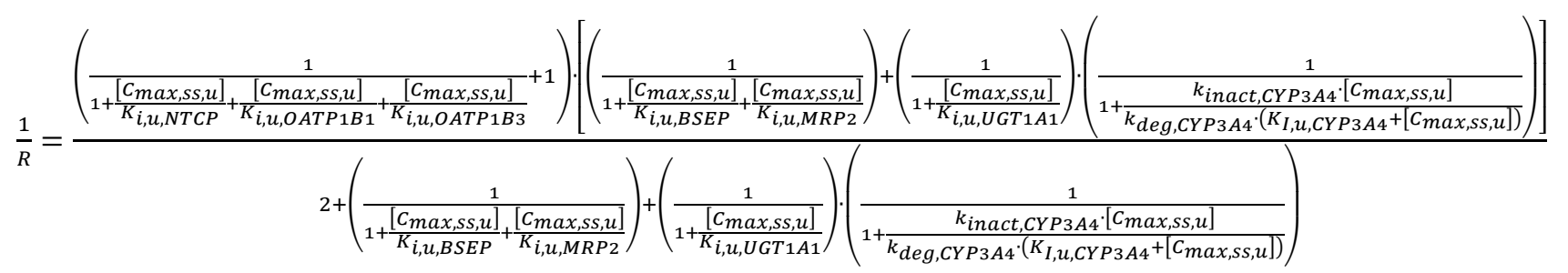

\subsection{Data Collection and Analysis}

To evaluate the ECM-based DILI potential in vitro inhibition $\left(\mathrm{K}_{\mathrm{i}, \mathrm{u}}, \mathrm{K}_{\mathrm{I}, \mathrm{u}}\right.$ and $\left.\mathrm{k}_{\text {inact }}\right)$ and in vivo exposure $\left(\mathrm{C}_{\mathrm{max}, \mathrm{ss}, \mathrm{u}}\right)$ data for 420 drug compounds were compiled (as available) from the literature (no additional data points were generated for the purpose of this work). As a basic principle, the most conservative (i.e. highest) reported concentration value, determined at the highest clinical dose, was used. In the absence of experimental confirmation $\mathrm{K}_{\mathrm{i}, \mathrm{u}}=\mathrm{K}_{\mathrm{i}}=\mathrm{IC}_{50}=\mathrm{IC}_{50, \mathrm{u}}$ and $\mathrm{K}_{\mathrm{I}, \mathrm{u}}=\mathrm{K}_{\mathrm{I}}$ was assumed. Microsoft EXCEL was used to calculate the $1 / \mathrm{R}$ values according to Eq. 5 for the entire dataset.

\section{Panel A}

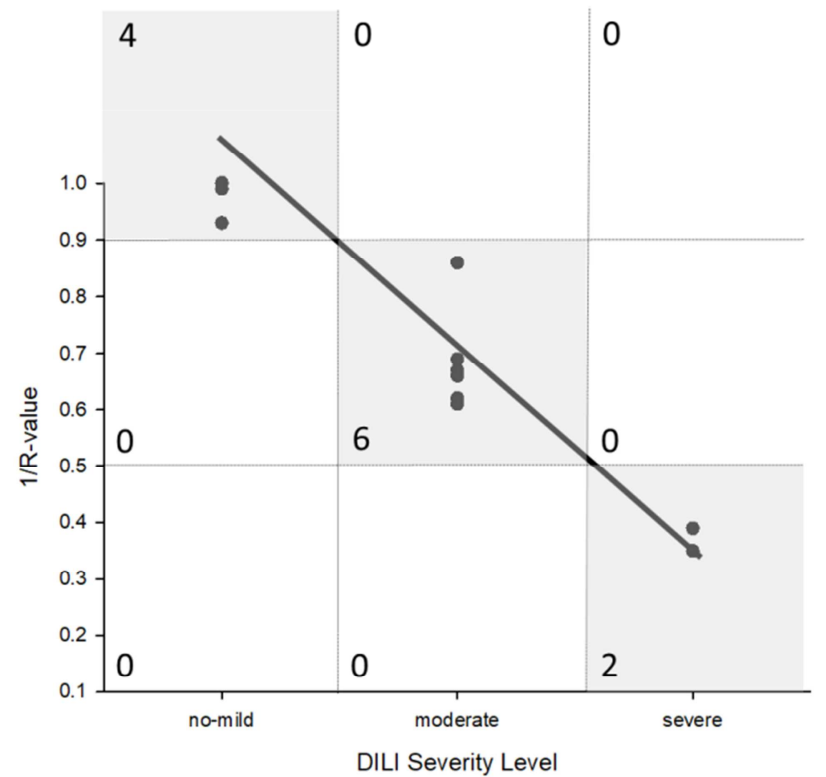

\section{Results}

Figure 1 (panel A) shows the relationship between 1/R-value according to Eq. 5 and DILI severity level for a limited but largely complete in-house dataset $(\mathrm{N}=12)$ (Table 2). In Figure 1 (panel B) the same 1/R vs DILI severity class relationship is shown for a subset of the FDA DILIrank database for which relevant exposure and inhibition information could be compiled from literature $(\mathrm{N}=408$ out of 1045) (Supplementary Table 1: https://figshare.com/articles/DILI Camenisch Supplementary Table) [9].

\section{Panel B}

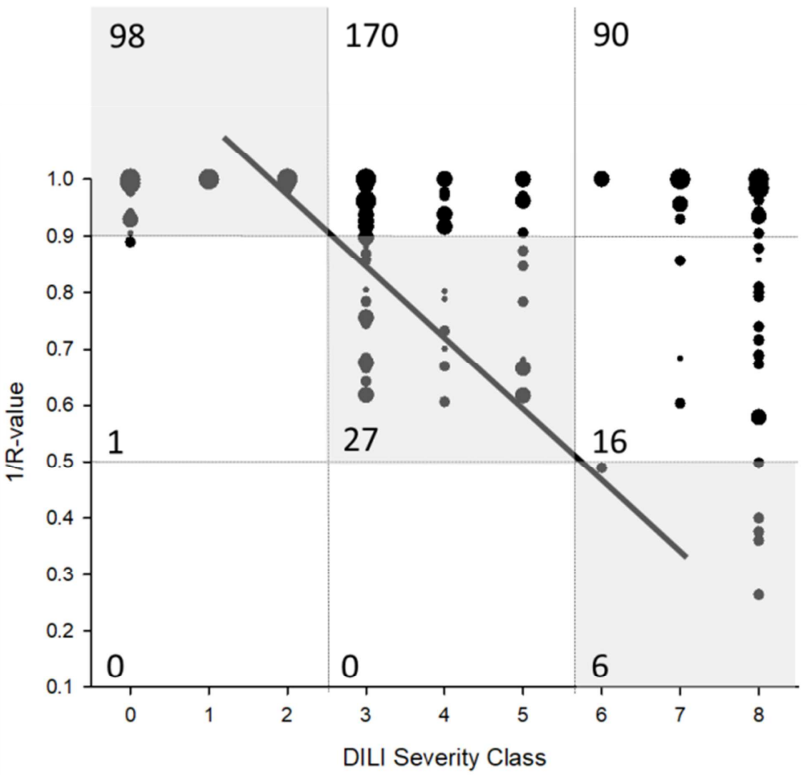

Figure 1. 1/R-value vs DILI severity relationships according to Eq. 5 as derived from inhibition, exposure and DILI severity data are summarized in Table 2 (panel A, in-house dataset) and Supplementary Table 1 (panel B, FDA DILIrank database). The horizontal dotted lines at 1/R-values of 0.9 and 0.5 represent the limits between no/mild to moderate and between moderate to severe DILI scores (vertical dotted lines) as suggested from panel A. Numbers in the grey highlighted areas refer to the sum of compounds correctly assigned to the right DILI severity level/class. Numbers in the non-colored areas above the vertical black line denote false negative predictions and the corresponding numbers below the vertical black line represent false positive DILI severity estimates. Bubble sizes in panel B refer to one of the four Biopharmaceutical Drug Disposition Classification System (BDDCS) classes (class one smallest, class four largest) [10].

Table 2. Experimental exposure and inhibition constants for a selected Novartis dataset.

\begin{tabular}{|c|c|c|c|c|c|c|c|c|c|c|c|}
\hline \multirow{2}{*}{$\begin{array}{l}\text { Cpd } \\
\text { name }\end{array}$} & \multirow{2}{*}{$\begin{array}{l}\text { DILI } \\
\text { severity } \\
\text { level }^{\text {a) }}\end{array}$} & \multirow{2}{*}{$\begin{array}{l}C_{\max , \mathrm{ss}, \mathrm{u}} \\
(\boldsymbol{\mu M})\end{array}$} & \multicolumn{2}{|c|}{ CYP3A4 } & $\begin{array}{l}\text { UGT1A } \\
1\end{array}$ & BSEP & MRP2 & OATP1B1 & OATP1B3 & NTCP & $1 / R^{b)}$ \\
\hline & & & $K_{I, u}(u M)$ & $k_{\text {inact }}\left(\min ^{-1}\right)$ & \multicolumn{7}{|c|}{$K_{i, u}(u M)$} \\
\hline Novartis 1 & moderate & 1.30 & 35 & 0.02 & ni & 4.7 & $\mathrm{nq}$ & 33.4 & $\mathrm{nr}$ & $\mathrm{ni}$ & 0.59 \\
\hline Novartis 2 & severe & 0.36 & 3.2 & 0.023 & $\mathrm{nr}$ & 1.6 & 5.9 & 0.7 & 0.7 & 1 & 0.44 \\
\hline Novartis 3 & moderate & 0.12 & $\mathrm{nr}$ & $\mathrm{nr}$ & 0.05 & ni & ni & 1.1 & 5.6 & 20 & 0.74 \\
\hline Novartis 4 & severe & 1.01 & 13.6 & 0.026 & 0.09 & 9.1 & ni & 1.7 & 2.4 & ni & 0.47 \\
\hline Novartis 5 & moderate & 0.05 & 0.5 & 0.081 & ni & ni & $\mathrm{nr}$ & 5 & 5 & ni & 0.68 \\
\hline Novartis 7 & no-mild & 0.13 & $\mathrm{nr}$ & $\mathrm{nr}$ & ni & $\mathrm{nr}$ & $\mathrm{nr}$ & 1.79 & 13.76 & ni & 0.96 \\
\hline
\end{tabular}




\begin{tabular}{|c|c|c|c|c|c|c|c|c|c|c|c|}
\hline \multirow{2}{*}{$\begin{array}{l}\text { Cpd } \\
\text { name }\end{array}$} & \multirow{2}{*}{$\begin{array}{l}\text { DILI } \\
\text { severity }^{\text {level }}{ }^{\text {a) }} \\
\end{array}$} & \multirow{2}{*}{$\begin{array}{l}C_{\text {max,ss,u }} \\
(\mu \mathrm{M})\end{array}$} & \multicolumn{2}{|c|}{ CYP3A4 } & $\begin{array}{l}\text { UGT1A } \\
1\end{array}$ & BSEP & MRP2 & OATP1B1 & OATP1B3 & NTCP & $1 / R^{b)}$ \\
\hline & & & $\mathrm{K}_{\mathrm{I}, \mathrm{u}}(\mathrm{uM})$ & $k_{\text {inact }}\left(\min ^{-1}\right)$ & \multicolumn{7}{|c|}{$\mathbf{K}_{\mathrm{i}, \mathrm{u}}(\mathbf{u M})$} \\
\hline Novartis 8 & no-mild & 0.06 & 66.0 & 0.03 & ni & 78.2 & $\mathrm{nr}$ & 27.3 & 25.2 & ni & 0.98 \\
\hline Novartis 9 & moderate & 0.59 & $\mathrm{nr}$ & $\mathrm{nr}$ & 0.77 & 6.3 & $\mathrm{nr}$ & 6.5 & 17 & ni & 0.8 \\
\hline Novartis 10 & no-mild & 0.05 & $\mathrm{nr}$ & $\mathrm{nr}$ & ni & 202 & 78 & 136 & 3.9 & 50 & 0.99 \\
\hline Novartis 11 & no-mild & 0.05 & 14.5 & 0.005 & ni & $\mathrm{nr}$ & $\mathrm{nr}$ & $\mathrm{nr}$ & $\mathrm{nr}$ & ni & 0.99 \\
\hline Novartis 12 & no-mild & 0.0002 & $\mathrm{nr}$ & $\mathrm{nr}$ & ni & $\mathrm{nr}$ & 74.5 & 69.9 & $\mathrm{nr}$ & ni & 0.99 \\
\hline
\end{tabular}

nr: negative or irrelevant experimental outcome

ni: absence of experimental data or measured inhibition constants

a) Only compounds with likely transporter- and/or enzyme-derived cell stress mechanism explanation were included

b) Calculated according to Eq. 5 with $\mathrm{k}_{\mathrm{deg}, \mathrm{CYP} 3 \mathrm{~A} 4}$ equal to $0.000321 \mathrm{~min}^{-1}$ [6]. For any missing inhibition information the $\mathrm{CL}_{\text {int, }} / \mathrm{CL}_{\text {int }}$ ratio calculation according to Eq. 2 was fixed, in accordance with lack of process inhibition, at a value of 1

\section{Discussion}

In the present work, the use of the Extended Clearance Model for DILI risk assessment upon designated transporterand enzyme- process inhibition is investigated. Using integrated ratios between systemic drug concentrations and inhibition constants (so-called 1/R-values), an obvious linear relationship with the DILI severity determined in clinics is established. The lower the 1/R-value the more likely a relevant transporter- and/or enzyme-derived cell stress event will occur. As such, a nice separation between the different DILI severity levels (no-mild $(1 / \mathrm{R}>0.9)$, moderate $(0.9>1 / \mathrm{R}>0.5)$, severe $(1 / \mathrm{R}<0.5))$ is achieved as demonstrated by the largely comprehensive but small set of in-house compounds (Figure 1, panel A). The same breakup, although being associated with a large proportion of predominantly false negative DILI predictions (about $90 \%$ for all compounds assigned to the moderate and severe DILI level groups), is also seen for the extensive FDA DILIrank dataset (Figure 1, panel B). However, taking into consideration that the latter relationship referrs to a mainly incomplete dataset (solely 6 cpds with complete data information) and that the current assessment is limited to process inhibition mechanisms already confirmed to be associated with DILI (Eq. 5) this is not unexpected. Moreover, as shown elsewhere, to assess the actual intrahepatic inhibition potential properly the use of unbound systemic drug concentrations is predominantly justified for ECM class 2 cpds for which $\mathrm{CL}_{\mathrm{sec}}$ and $\mathrm{CL}_{\text {met }}$ are the rate limiting intrinsic hepatic clearance processes (i.e. if $\mathrm{PS}_{\text {inf }}=\mathrm{PS}_{\text {eff }}>$ $\mathrm{CL}_{\mathrm{sec}}+\mathrm{CL}_{\text {met }}$ ) [8]. For all other ECM classes (namely 1, 3 and 4 ), as the sinusoidal membrane might act as a transport barrier limiting access to the hepatocytes, the use of $\mathrm{C}_{\max , \mathrm{ss}, \mathrm{u}}$ might result in an under- or over-estimation of intrahepatic process inhibition and consequently of the $1 /$ R-value. As such, in line with previous observations, it is it not astonishing that predominantly highly permeable compounds have been associated with increased DILI risk (in the context of BDDCS mainly class 1 and 2 cpds (Figure 1 (panel B)) [10]).

In the face of rising drug development costs and regulatory demands, the obvious difficulty researchers are facing is that they cannot prospectively anticipate the DILI potential of drug candidates. Referring to Eq. 5 it is obvious that significant inhibition of a single pathway or process will maximally result in a moderate DILI. On the other hand, severe transporterand/or enzyme-derived cell stress only occurs if more than one hepatic intrinsic clearance pathway is considerably impacted in parallel. Consequently, despite all of the simplifications and approximations applied (e.g. known rescue mechanisms, e.g. via sinusoidal efflux transporters MRP3 or MRP4, have been deliberately been factored out), the $1 / \mathrm{R}$-value approach provides a numerical representation of the overall dose-dependent transporter- and/or enzyme-derived cell stress of new chemical entities. Hence, the underlying data used for the present work is unquestionably subject to exhaustive incompleteness, inherent variability (e.g. $\mathrm{C}_{\text {max,ss,u }}$ ), uncertainty (e.g. $\mathrm{K}_{\mathrm{i}}$ vs $\mathrm{K}_{\mathrm{i}, \mathrm{u}}$ vs $\mathrm{IC}_{50}$ or unbound systemic vs unbound intrahepatic concentration), inconsistency (e.g. assessment of ultimate maximal daily dose), bias (e.g. for some drugs DILI is caused by a metabolite rather than parent compound), and/or impartiality (i.e. for the DILIrank dataset no preselection based on underlying DILI mechanism was done). As such, perfect quantitative $1 / \mathrm{R}$ vs DILI severity projections are, as yet, unrealistic.

\section{Conclusion}

Many approaches to anticipate the DILI potential of drug candidates have been described in the literature $[5,6]$. However, most (if not all) attempts concentrate on a single mechanism or on empirical qualitative binning methodologies if combinations of approaches are assessed [4]. In contrast, the ECM-derived 1/R-value approach presented here denotes a model-based approach to anticipate the cell stress derived DILI risk in an integral (all currently known enzyme- and transporter-derived mechanisms have been incorporated in the model) and quantitative (the lower the $1 / \mathrm{R}$-value determined for a drug candidate the higher is its likelihood to cause DILI) manner. Referring to a largely complete in-house dataset perfect DILI anticipation was achieved. However, the prediction accuracy practically collapses when the required input information is largely incomplete.

Nevertheless, the existing analysis is very encouraging and certainly worth further research. Hence, this early work indicates the beginning of a new avenue. The observed relationships certainly need to be validated (and likely adjusted) with a more comprehensive data set. To expand further our (mechanistic) understanding and to improve our 
prediction confidence widened and coordinated data access is urgently needed. Moving forward we will require improved access to high-quality in vitro process inhibition data, enhanced availability of relevant in vivo exposure information and better transparency concerning clinical DILI manifestation/scoring among the industry. Via collaborations (e.g. in form of consortia) synergies could be created and existing knowledge certainly could be leveraged. Enlarged data sharing will ultimately enable machine-learning opportunities and therefore the future development of analytical and computational tools in support of DILI prediction efforts (e.g. cheminformatics). Hence, a much better partnership between the key players involved in healthcare research is herewith encouraged.

\section{References}

[1] S. Russmann, G. Kullak-Ublick, and I. Grattagliano (2009). Current concepts of mechanisms in drug-induced hepatotoxicity. Curr Med Chem 16, 3041-53.

[2] M. Chen, V. Vijay, Q. Shi, Z. Liu, H. Fang, and W. Tong (2011). FDA-approved drug labeling for the study of drug-induced liver injury. Drug Discov Today 16, 697-703.

[3] Y. Shitara, H. Sato, and Y. Sugiyama (2005). Evaluation of drug-drug interaction in the hepatobiliary and renal transport of drugs. Annu Rev Pharmacol Toxicol 45, 689-723.
[4] S. Schadt, S. Simon, S. Kustermann, et al. (2015) Minimizing DILI risk in drug discovery - a screening tool for drug candidates. Toxicology In Vitro 30, 429-437.

[5] J. Chang, E. Plise, J. Cheong, Q. Ho, and M. Lin (2013). Evaluating the in vitro inhibition of UGT1A1, OATP1B1, OATP1B3, MRP2 and BSEP in predicting drug-induced hyperbilirubinemia. Mol Pharmaceutics 10, 3067-3075.

[6] O. Fahmi, T. Maurer, M. Kish, E. Cardenas, S. Boldt, and D. Nettleton (2008). A combined model for predicting CYP3A4 clinical net drug-drug interaction based on CYP3A4 inhibition, inactivation, and induction determined in vitro. Drug Metab Dipos 36, 1698-1708.

[7] G. Camenisch, and K. Umehara (2012). Predicting human hepatic clearance from in vitro drug metabolism and transport data: A scientific and pharmaceutical perspective for assessing drug-drug interactions. Biopharm Drug Dispos 33, 179-194.

[8] G. Camenisch, J. Riede, A. Kunze, J. Huwyler, B. Poller, and K. Umehara (2015). The extended clearance model and its use for the interpretation of hepatobiliary elimination data. ADMT\&DMPK 3, 1-14.

[9] M. Chen, A. Suzuki, S. Thakkar, K. Yu, C. Hu, and W. Tong (2016). DILIrank: the largest reference drug list ranked by the risk for developing drug-induced liver injury in humans. Drug Discov Today 21, 648-653.

[10] R. Chan, and L. Benet (2017). Evaluation of DILI predictive hypothesis in early drug development. Chem Res Toxicol 30, 1017-1029. 